\title{
Intersession reliability of a posturo-stabilometric test, using a force platform
}

\author{
Alberto Baldini ${ }^{\mathrm{a}, *}$, Alessandro Nota ${ }^{\mathrm{a}}$, Valentina Assi ${ }^{\mathrm{b}}$, Fabiana Ballanti ${ }^{\mathrm{a}}$, Paola Cozza ${ }^{\mathrm{a}}$ \\ a Department of Orthodontics, University of Rome "Tor Vergata", Italy \\ ${ }^{\mathrm{b}}$ Wolfson Istitute of Preventive Medicine, Queen Mary University of London, United Kingdom
}

\section{A R T I C L E I N F O}

\section{Article history:}

Received 20 March 2013

Received in revised form 3 August 2013

Accepted 6 August 2013

Available online $\mathrm{xxxx}$

\section{Keywords:}

Posture

Postural balance

Reproducibility of results

Dental occlusion

\begin{abstract}
A B S T R A C T
Aim of the study: To evaluate the intersession reliability of a posturo-stabilometric examination. Methods: Single blind clinical trial conducted in two sessions over two weeks.

44 healthy volunteers free from postural and temporomandibular disorders. All the subjects complied with the criteria for completing the study.

All the subjects underwent two sessions of posturo-stabilometric examinations in different visual and mandibular conditions.

Sway area, sway length and the coordinates of the center of pressure were evaluated and statistically analyzed using the Intraclass correlation coefficient (ICC).

Results: All the posturo-stabilometric parameters seemed to have an excellent reproducibility with overall ICCs higher than $70 \%$ and good confidence intervals except for the sway area (ICC 0.422 with $\mathrm{CI} 0.283-$ 0.560 with open eyes and ICC 0.554 with CI $0.424-0.683$ with closed eyes).

Conclusions: The posturo-stabilometric examination carried out using a force platform has a good intrasession and intersession reliability, especially considering sway velocity, COP X and COP $Y$ parameters. The force platform usefulness in analyzing static posture is confirmed in any medical field.
\end{abstract}

(c) 2013 Elsevier Ltd. All rights reserved.

\section{Introduction}

Postural control and stability are primary components in maintaining the upright position and the equilibrium during daily movements and activities and have also important implications in sports medicine and rehabilitation.

There are many methods to assess postural control (Chaudhry et al., 2004; Pierchala et al., 2012), one of the most used is the study of the body center of pressure (COP) oscillations using a force platform (Baldini, 2010; Lanska, 2001) and the use of the stabilometric platform in order to evaluate posture is a standard for both clinical practice and research in many medical fields (Baldini et al., 2011, 2013a,b; Baldini and Cravino, 2011).

In fact the COP alterations are proportional to ankle torque, a combination of descending motor commands as well as mechanical properties of the surrounding musculature (Baratto et al., 2002). The most commonly evaluated parameters are sway length/velocity and area traversed based upon sequential locations of the COP in the plane of the force platform, and the displacement

\footnotetext{
* Corresponding author. Address: Centro Medico Polispecialistico Baldini Srl, Via S. Orsola, n. 5, Bergamo 24048, Italy. Tel.: +39 035 271935; fax: +39 035694280.

E-mail address: studiomedicobaldini@gmail.com (A. Baldini).
}

of the COP mean location on the $X$ and $Y$ axis (Gangloff et al., 2000; Manfredini et al., 2012).

This approach is used in dentistry as well, as part of gnathological therapies (Baldini et al., 2011, 2012a,b) and to investigate the potential associations between dental occlusion and body posture (Baldini et al., 2013a,b; Baldini and Cravino, 2011; Manfredini et al., 2012). These associations are the subject of an ongoing discussion as evidence has been published both in support of their existence (Bracco et al., 2004; Manfredini et al., 2012; Sakaguchi et al., 2007) and in disagreement with it (Michelotti et al., 2006; Perinetti, 2006). Moreover some criticism was made against the use of stabilometric platform as a trustworthy instrument. In fact in the literature review by Perinetti and Contardo (2009) it is concluded that although an association between the masticatory system and body posture probably exists, its clinical significance is low and a force platform is not a suitable instrument in dental diagnosis.

Some authors (Morasso et al., 2002; Perinetti, 2006) criticized the results of the posturo-stabilometric examination and their clinical implications because of the lack of studies demonstrating the scientific trustworthiness of the force platform. This observation could discredit every result obtained so far using this instrument in any field of medicine as well as what concerns the correlation between dental occlusion and posture. These remarks indicate 
the urgent need of assessing the reliability of this examination and also to confirm the validity of this diagnostic instrument in any related medical field. Once the reliability of the examination has been ascertained, it will be possible to verify the existence and degree of association between occlusion and posture.

The accuracy and precision of the instrument should be guaranteed by the producer himself and by the maintenance and calibration of the device. However, he is not responsible for the reproducibility of the test and the effective utility and benefit for a gnathologist who applies it to show occlusal interferences over posture.

There are three types of random statistical variability concerning the reliability of the examinations: the intrasession variability that is related to the immediate retest reliability, the intersession variability, related to delayed retest reliability, and the interrater variability, related to delayed retest reliability with different operators (Bauer et al., 2010).

A survey of the PubMed MEDLINE database and a review article (Ruhe et al., 2010) shows that some articles (Corriveau et al., 2000; Lafond et al., 2004; Lin et al., 2008; Salavati et al., 2009; Santos et al., 2008) assessed the reliability of the force plate examination, considering only the intrasession variability, obtaining excellent results. The intersession reliability was the subject of a recent study by Perinetti et al. (2012).

An experimental protocol has been created in order to evaluate the intersession variability of the force plate examination. The aim of this study is to assess the reproducibility of this examination with important influences in research and even more in clinical practice, as it could allow us to verify the effects of the therapeutic plan in the short or long term and thus monitor the postural modifications of the patient.

\section{Methods}

\subsection{Data collection}

Forty-four healthy subjects, 30 males and 14 females (age $17-35$ years, mean age $23.75 \pm 4.10$ years) were enrolled in the study after signing an informed consent form.

Every subject underwent an occlusal (Baldini et al., 2009), postural and anamnestic examination to verify the compliance with the following inclusion criteria:

- Good general health according to medical history.

- Absence of trauma or surgery, which could influence posture.

- Absence of visual or vestibular problems.

- Absence of any other disorder able to influence posture.

- Absence of evident postural problems.

- Presence of not less than 28 teeth.

- Dental overjet between 1 and $4 \mathrm{~mm}$.

- Absence of any type of crossbite, open bite or deep bite.

- Absence of cast restorations and extensive occlusal restoration.

- Absence of temporomandibular disorders (TMD).

This single blind experimental protocol was carried out using a force platform Postural Health Station (DL Medica S.p.A. Milano, Italy) (Fig. 1), and the ethics committee approved the study.

\subsection{Test}

All subjects were volunteers with no information about the aim of the study and who underwent a posturo-stabilometric force platform examination. Each recording lasted $51.2 \mathrm{~s}$ under the following conditions: mandibular rest position, mandibular position of centric occlusion and mandibular position with cotton rolls.

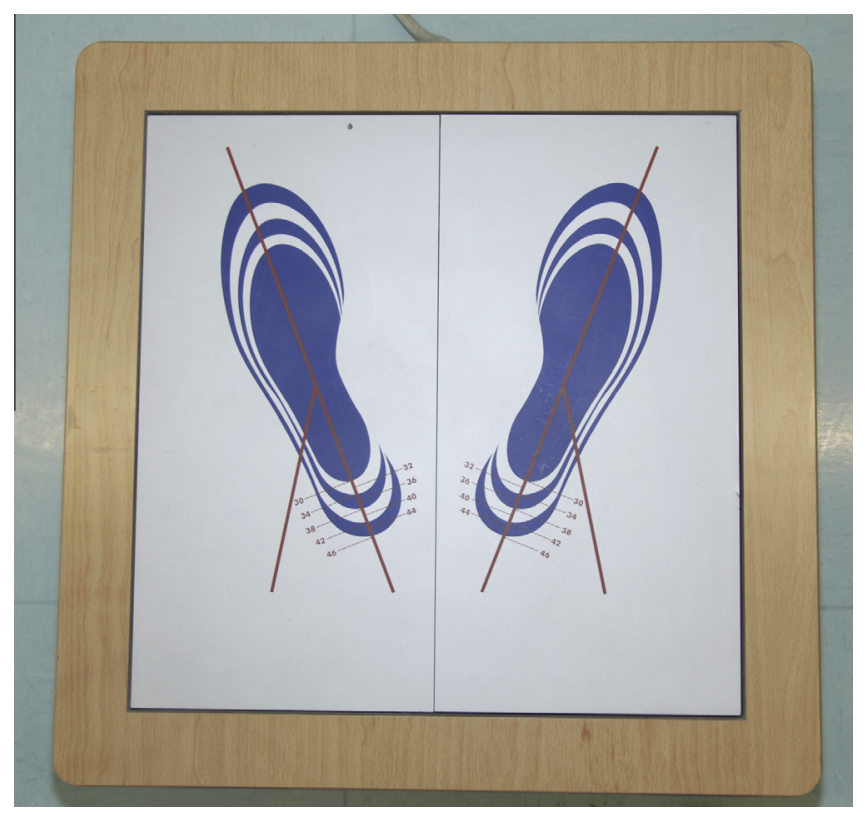

Fig. 1. Posturo-stabilometric platform Postural Health Station (DL Medica S.p.A. Milano, Italy).

The test was repeated (twice) in these three positions with both eyes open and closed. For the cotton rolls position, cotton rolls $8 \mathrm{~mm}$ thick and $37 \mathrm{~mm}$ long were positioned on the mandibular teeth distal to the canines.

Quiet conditions were maintained during the examination, thus eliminating any disturbing element that could affect the posture. A force plate was placed in order to position subjects perpendicular to and facing the wall at $150 \mathrm{~cm}$. The subjects were required to remain as stable as possible, relaxed, with their arms hanging free beside their trunk, and facing the wall without concentrating on a precise point on it. They were asked to avoid alcohol, sport and conservative dental therapies during the $24 \mathrm{~h}$ before the clinical recordings.

The standardized placement of the subjects on the force plate is fundamental to reduce the intersession variability of the examination: a hand was placed under the foot of the subject, lifting the foot until it reached the following criteria using the markers painted on the surface of the platform: (Fig. 1)

- Feet angle of $30^{\circ}$ following the principal red line.

- Calcaneal tendon positioned in correspondence of the length of the foot, expressed in French points and centered on the principal red line.

- Malleolus positioned in correspondence of the angled red line.

- Second foot finger root projection correspondent to the principal red line.

- Foot outline correspondent to the areas drawn on the surface of the platform.

Every subject underwent two examination sessions,with a pause of 14 days. The two sessions were conducted following the same modalities and with conditions as identical as possible, to allow the study of intersession reliability. In order to exclude the potential "interrater variability", all tests were performed by the same operator.

In order to be included, it was required that the subjects satisfy the following criteria in the 14 days prior the second session of tests:

- Absence of any trauma.

- Absence of restorative or orthodontic treatment. 
- Absence of any pain that could influence posture.

All the subjects in the sample satisfied these second phase criteria.

From the results obtained four posturo-stabilometric parameters were evaluated:

- SWAY AREA.

- SWAY LENGTH.

- COP $X$ (position of the center of foot pressure on the $X$ axis compared to the theoretical one).

- COP Y (position of the center of foot pressure on the $Y$ axis compared to the theoretical one).

\subsection{Statistical analyses}

The distribution of the posturo-stabilometric parameters recorded, sway area and length and coordinates of the center of foot pressure, were summarized as means and SDs, according to test position, i.e. at mandibular rest position, mandibular position of centric occlusion and mandibular position with cotton rolls to whether eyes were open or closed and to the session of examinationination. Wilcoxon matched-pair signed-rank test was used to verify the hypothesis of a non-significant difference in parameters between sessions.

One of the aims of this study is to evaluate the reliability of the posturo-stabilometric test, i.e. whether the resulting measurements of this test are reproducible among sessions. As it was reasonable to believe that no systematic error occurred, the intraclass correlation coefficient (ICC) was estimated to ensure test-retest reliability between sessions (Rousson et al., 2002) ICC may be defined as the percentage of variability in measurements explained by the subject effect thus the larger this percentage the better the reliability. An ICC larger than 0.75 indicates good to excellent reliability, an ICC included between 0.40 and 0.75 indicates fair to good reliability, and finally an ICC lower than 0.40 indicates poor reliability (Corriveau et al., 2000).

Therefore, in order to ensure the reproducibility of test results between sessions, ICCs were computed for each posturo-stabilometric parameter according to both test and eye position. ANOVA was then run separately for open and closed eyes to verify whether the distributions of the parameters were similar or not, despite the test position. We then merged the observations for the parameters and hence compared, through ICC, six repetitions of the test, instead of two.

All analyses were performed with STATA software version 12.1.

\section{Results}

A total of 44 subjects were included in the study and underwent the posturo-stabilometric test twice, within a two-week interval. Descriptive statistics of the results of the examinations are summarized in Table 1. In general the time difference between test sessions did not affect the measures of the posturographic parameters and the Wilcoxon matched-pair signed-rank tests led us to accept the hypothesis of no change in most cases. The only significant difference between sessions was observed for the sway area measurements assessed in mandibular position with cotton rolls and eyes open.

The reliability was investigated estimating ICCs for every position with eyes open and closed (Table 2). Generally, ICCs for sway length and coordinates of the center of foot pressure were over $70 \%$, confirming the repeatability of the test. Sway area, on the other hand, performed poorly, even obtaining an ICC of $37 \%$

Table 1

Descriptive statistics for the posturographic parameters at the two examination sessions according to the position.

\begin{tabular}{|c|c|c|c|c|c|}
\hline test & First session & & Second session & & $p$-value \\
\hline \multicolumn{6}{|c|}{ Mean (SD) } \\
\hline \multicolumn{6}{|c|}{ Eyes open } \\
\hline \multicolumn{6}{|c|}{ Rest position } \\
\hline Area & 94.25 & $(43.48)$ & 104.17 & $(56.37)$ & .155 \\
\hline Length & 293.85 & $(68.31)$ & 304.91 & $(94.24)$ & .591 \\
\hline $\operatorname{COP} X$ & 1.40 & $(7.62)$ & 1.05 & $(8.01)$ & .434 \\
\hline COP Y & -8.12 & $(9.96)$ & -5.95 & (11.67) & .059 \\
\hline \multicolumn{6}{|c|}{ Centric occlusion } \\
\hline Area & 107.97 & $(62.95)$ & 113.82 & $(65.51)$ & .944 \\
\hline Length & 295.34 & (69.37) & 287.55 & $(76.74)$ & .414 \\
\hline COP $X$ & 1.06 & $(8.02)$ & 1.93 & $(7.98)$ & .220 \\
\hline COP Y & -7.02 & $(12.20)$ & -6.13 & $(12.76)$ & .815 \\
\hline \multicolumn{6}{|c|}{ Cotton rolls position } \\
\hline area & 109.68 & $(80.57)$ & 122.67 & $(71.83)$ & $.034^{*}$ \\
\hline length & 296.03 & $(78.34)$ & 306.93 & $(85.31)$ & .059 \\
\hline COP $X$ & 2.27 & $(7.61)$ & 1.71 & $(7.73)$ & .963 \\
\hline COP $Y$ & -8.05 & $(11.62)$ & -8.51 & $(12.06)$ & .608 \\
\hline \multicolumn{6}{|c|}{ Eyes closed } \\
\hline \multicolumn{6}{|c|}{ Rest position } \\
\hline Area & 143.75 & (94.08) & 154.31 & (123.89) & .462 \\
\hline Length & 419.86 & $(122.13)$ & 405.23 & $(125.77)$ & .072 \\
\hline $\operatorname{COP} X$ & 2.20 & (7.51) & 1.19 & $(7.45)$ & .062 \\
\hline COP Y & -6.64 & $(10.49)$ & -5.94 & $(10.44)$ & .477 \\
\hline \multicolumn{6}{|c|}{ Centric occlusion } \\
\hline Area & 172.34 & $(130.03)$ & 186.61 & $(152.48)$ & .889 \\
\hline Length & 416.53 & $(134.40)$ & 405.75 & (121.65) & .273 \\
\hline COP $X$ & 1.84 & $(8.51)$ & 1.63 & (7.29) & .591 \\
\hline COP Y & -9.18 & $(11.32)$ & -8.77 & $(12.17)$ & .815 \\
\hline \multicolumn{6}{|c|}{ Cotton rolls position } \\
\hline Area & 177.63 & $(143.59)$ & 195.99 & $(227.62)$ & .981 \\
\hline Length & 408.01 & $(127.72)$ & 406.94 & (127.17) & .554 \\
\hline COP $X$ & 2.06 & $(6.81)$ & 1.29 & (7.27) & .491 \\
\hline COP Y & -9.80 & (11.19) & 10.02 & $(11.44)$ & .692 \\
\hline
\end{tabular}


Table 2

The repeatability of the posturographic parameters for the different recording conditions assessed with intraclass correlation coefficients (ICCs).

\begin{tabular}{|c|c|c|c|c|c|c|}
\hline \multirow[t]{2}{*}{ Variable } & \multicolumn{3}{|c|}{ Eyes open } & \multicolumn{3}{|c|}{ Eyes closed } \\
\hline & ICC & [95\% Conf] & [Interval] & ICC & [95\% Conf] & [Interval] \\
\hline \multicolumn{7}{|c|}{ Rest position } \\
\hline Area & 0.553 & 0.347 & 0.759 & 0.560 & 0.356 & 0.764 \\
\hline Length & 0.768 & 0.647 & 0.890 & 0.773 & 0.653 & 0.893 \\
\hline $\operatorname{COP} X$ & 0.799 & 0.691 & 0.906 & 0.823 & 0.727 & 0.919 \\
\hline COP Y & 0.608 & 0.420 & 0.795 & 0.660 & 0.492 & 0.827 \\
\hline \multicolumn{7}{|c|}{ Centric occlusion } \\
\hline Area & 0.457 & 0.221 & 0.692 & 0.687 & 0.530 & 0.844 \\
\hline Length & 0.731 & 0.592 & 0.869 & 0.796 & 0.688 & 0.905 \\
\hline $\operatorname{COP} X$ & 0.758 & 0.631 & 0.884 & 0.764 & 0.640 & 0.888 \\
\hline COP Y & 0.792 & 0.681 & 0.903 & 0.787 & 0.674 & 0.900 \\
\hline \multicolumn{7}{|c|}{ Cotton rolls position } \\
\hline Area & 0.371 & 0.114 & 0.627 & 0.440 & 0.200 & 0.679 \\
\hline Length & 0.811 & 0.709 & 0.913 & 0.738 & 0.601 & 0.874 \\
\hline COP $X$ & 0.804 & 0.698 & 0.910 & 0.798 & 0.691 & 0.906 \\
\hline COP $Y$ & 0.805 & 0.700 & 0.909 & 0.738 & 0.603 & 0.873 \\
\hline \multicolumn{7}{|c|}{ Overall (6 repetitions) } \\
\hline Area & 0.422 & 0.283 & 0.560 & 0.554 & 0.424 & 0.683 \\
\hline Length & 0.734 & 0.638 & 0.829 & 0.780 & 0.696 & 0.863 \\
\hline COP $X$ & 0.759 & 0.670 & 0.848 & 0.758 & 0.669 & 0.848 \\
\hline COP Y & 0.661 & 0.548 & 0.773 & 0.714 & 0.614 & 0.815 \\
\hline
\end{tabular}

Table 3

$P$-values for $F$-test verifying null hypothesis of no change in distribution according to recording position.

\begin{tabular}{lll}
\hline \multirow{2}{*}{ Parameter } & \multicolumn{2}{l}{ Prob $>F$} \\
\cline { 2 - 3 } & Eyes open & Eyes closed \\
\hline Area & .203 & .211 \\
Length & .674 & .963 \\
COP $X$ & .862 & .998 \\
COP $Y$ & .608 & .083 \\
\hline
\end{tabular}

in mandibular position with cotton rolls and eyes open, as we might expect after the previous Wilcoxon test result. In the same recording position the other posturo-stabilometric parameters seemed to have an excellent reproducibility with ICCs higher than $80 \%$. Moreover, ICCs for sway area were also consistently higher when the test was performed with eyes closed.

Overall ICCs (Table 2) were estimated after results from the F-test in ANOVA (Table 3) confirmed that the posturo-stabilometric parameters were distributed in a similar way in all the three positions, i.e. mandibular rest position, mandibular position of centric occlusion, and mandibular position with cotton rolls, after separating for eyes open or closed. These appeared to mirror the results in the three test positions, with good reproducibility for sway length and coordinates of the center of foot pressure. ICCs were generally over $70 \%$, especially with eyes closed. However, the results regarding the sway area confirmed the poor test-retest reliability previously observed.

\section{Discussion}

The study of the ICCs on four posturo-stabilometric parameters in six different mandibular/visual conditions calculated on two distanced sessions, demonstrated that the examination with the force plate not only has a good intrasession reliability, as Bauer et al. (2010), Ruhe et al. (2010), Corriveau et al. (2000) said, but also has a good intersession reliability.
Previous literature on intrasession variability (Bauer et al. 2010; Ruhe et al., 2010), focused only on the precision and the accuracy of the instrument and the variation of the phenomena, suggesting the utility of the instrument in a diagnostic setting but limited to a single test session. It is still not clear if this instrument could be useful during the follow-up analysis of a therapeutic plan. In fact, this test can easily be susceptible to intersession variability due to the different placement of the patient on the platform between different sessions or to the conditions of the patient, which can affect posture and load distribution.

The analysis of the intersession variability shows that reliability increases when subjects undergo the test with eyes closed. All the parameters showed an excellent reliability except for the sway area and there is no evident difference among values of ICC for the other parameters. The sway area appeared more reliable with eyes closed and when the mandible is positioned in centric occlusion and less reliable when cotton rolls are positioned between the dental arches. This may well be due to the higher sensibility of this parameter to occlusal modifications (Baldini et al., 2013a,b; Baldini and Cravino, 2011).

Perinetti et al. (2012) analyzed the intersession reliability after 30 min, 1 day and 7 days from the first test, and found that sway area had a good reliability and COP $X$ and COP $Y$ to be poorly reliable, contrarily to our results. The sway velocity in this study appeared to be a reliable parameter according to the ICC values for the sway length. Sway velocity is easily obtained by dividing the sway length by the time duration of the test and can thus be considered equal to the sway length. The results of Perinetti's and colleagues' study had low significance because of the limited size of their sample, that led to very wide confidence intervals for the ICCs of the coordinates of the center of foot pressure. This undermines the reproducibility of their results on a larger population.

Furthermore, the reliability of these parameters could be strictly associated to the rigorous rules and surface details for placement of the subject on the force platform.

Our findings support the results of the studies on intrasession reliability although it seemed that the sway area was the only parameter that looses reliability over time. However, asking the patients to maintain the eyes closed during this examination could be useful for increasing its reliability and should therefore be recommended for monitoring results in a therapeutic plan.

The sway area did not seem an ideal parameter for monitoring the evolution of a therapeutic plan. When it is necessary to consider the area of sway it is advisable to use the mandibular position of centric occlusion for better reliability in the results.

Our sample did not include subjects with temporomandibular and occlusal disorders and, currently, in the literature their influence on posture has yet to be clearly proved. Nevertheless, when a posture professional examines subjects affected by these disorders, the rest position may be preferred over the centric occlusion mandibular one to eliminate the temporo-occlusal interference and its possible influence on the reliability of the observations. This remark should be remembered until the reliability on a sample affected by temporomandibular disorders is assessed.

Even if some studies (Baldini et al., 2013a,b; Baldini and Cravino, 2011; Michelotti et al., 2006) demonstrated that the sway area is the most sensitive parameter for evaluating the correlation between dental occlusion and posture, the gnathologist should remember that this parameter is poorly reliable when the examination is carried out in the cotton rolls position and thus its use should probably be limited to diagnostic considerations in a single session and not within a longer therapeutic plan.

In agreement with what is said by Perinetti et al. (2012), given that dental occlusion causes statistically significant but low variations on the sway area and that in rest or centric occlusion 
mandibular positions its reliability improves, its usefulness in following a gnathological therapeutic plan should be investigated further.

\section{Conclusions}

The posturo-stabilometric examination carried out using a force platform has a good intrasession and intersession reliability, especially considering sway length, COP $X$ and COP $Y$ parameters. The force platform usefulness in analyzing static posture is confirmed in any medical field. The trustworthiness of this instrument for clinical gnathological purposes should be considered with caution owing to the "fair to good" reliability of the sway area parameter.

\section{References}

Baldini A. Clinical and instrumental treatment of a patient with dysfunction of the stomatognathic system: a case report. Ann Stomatol (Roma) 2010;1(2):2-5.

Baldini A, Beraldi A, Nanussi A. The clinical importance of computerized evaluation of occlusion. Dental Cadmos 2009;77(4):47-54

Baldini A, Cravino G, Rinaldi A, Cioffi D. Gnathological postural analysis and treatment in Air Force pilots: a case report. Mondo Ortodontico 2011;36(5):208-15.

Baldini A, Beraldi A, Nota A, Danelon F, Ballanti F, Longoni S. Gnathological postural treatment in a professional basketball player: a case report and an overview of the role of dental occlusion on performance. Ann Stomatol (Roma) 2012a:3(2):51-8.

Baldini A, Tecco S, Cioffi D, Rinaldi A, Longoni S. Gnathological postural treatment in an air force pilot. Aviat Space Environ Med 2012b;83(5):522-6.

Baldini A, Nota A, Cravino G, Cioffi C, Rinaldi A, Cozza P. Influence of vision an dental occlusion on body posture in pilots. Aviat Space Environ Med 2013a;84(8):823-7.

Baldini A, Nota A, Tripodi D, Longoni S, Cozza P. Evaluation of the correlation between dental occlusion and posture using a force platform. Clinics 2013b;68(1):45-9.

Baldini A, Cravino G. Dental occlusion and athletic performances. A review of literature Mondo Ortodontico 2011;36(3):131-41.

Baratto L, Morasso PG, Re C, Spada G. A new look at posturographic analysis in the clinical context: sway-density versus other parameterization techniques. Mot Control 2002;6(3):246-70.

Bauer C, Gröger I, Rupprecht R, Meichtry A, Tibesku CO, Gassmann KG. Reliability analysis of time series force plate data of community dwelling older adults. Arch Gerontol Geriatr 2010;51(3):e1005.

Bracco P, Deregibus A, Piscetta R. Effects of different jaw relations on postural stability in human subjects. Neurosci Lett 2004;356:228-30.

Chaudhry H, Findley T, Quigley KS, Bukiet B, Ji Z, Sims T, et al. Measures of postural stability. J Rehabil Res Dev 2004;41(5):713-20.

Corriveau H, Hebert R, Prince F, Raıche M. Intrasession reliability of the "center of pressure minus center of mass" variable of postural control in the healthy elderly. Arch Phys Med Rehabil 2000;81:45-8.

Gangloff P, Louis JP, Perrin PP. Dental occlusion modifies gaze and posture stabilization in human subjects. Neurosci Lett 2000;293(3):203-6.

Lafond D, Corriveau H, Hébert R, Prince F. Intrasession reliability of center of pressure measures of postural steadiness in healthy elderly people. Arch Phys Med Rehabil 2004;85(6):896-901.

Lanska DJ. Nineteenth-century contributions to the mechanical recording of postural sway. Arch Neurol 2001;58(7):1147-50.

Lin D, Seol H, Nussbaum MA, Madigan ML. Reliability of COP-based postural sway measures and age-related differences. Gait Posture 2008;28:337-42.

Manfredini D, Castroflorio T, Perinetti G, Guarda-Nardini L. Dental occlusion, body posture and temporomandibular disorders: where we are now and where we are heading for. J Oral Rehabil 2012;39(6):463-71.

Michelotti A, Buonocore G, Farella M, Pellegrino G, Piergentili C, Altobelli S, et al. Postural stability and unilateral posterior crossbite: is there a relationship? Neurosci Lett 2006;392(1-2):140-4.

Morasso P, Re C, Giacomozzi C, Macellari V. A testing device for the verification of the accuracy of the COP measurements in stabilometric platforms. Gait Posture 2002;16(Suppl. 1):S215-6.

Perinetti G. Dental occlusion and body posture: no detectable correlation. Gait Posture 2006;24(2):165-8.

Perinetti G, Contardo L. Posturography as diagnostic aid in dentistry: a Systematic review. J Oral Rehabil 2009;36(12):922-36.

Perinetti G, Marsi L, Castaldo A, Contardo L. Is postural platform suited to study correlations between the masticatory system and body posture? A study of repeatability and a meta-analysis of reported variations. Prog Orthod. 2012;13(3):273-80.

Pierchała K, Lachowska M, Morawski K, Niemczyk K. Sensory organization test outcomes in young, older and elderly healthy individuals - preliminary results. Otolaryngol Pol 2012;66(4):274-9.
Rousson V, Gasser T, Seifert B. Assessing intrarater, interrater and test-retest reliability of continuous measurements. Stat Med 2002;21(22):3431-46.

Ruhe A, Fejer R, Walker B. The test-retest reliability of centre of pressure measures in bipedal static task conditions - a systematic review of the literature. Gait Posture 2010;32(4):436-45.

Sakaguchi K, Mehta NR, Abdallah EF, Forgione AG, Hirayama H, Kawasaki T, et al. Examination of the relationship between mandibular position and body posture. Cranio 2007;25(4):237-49.

Salavati M, Hadian MR, Mazaheri M, et al. Test-retest reliability of center of pressure measures of postural stability during quiet standing in a group with musculoskeletal disorders consisting of low back pain, anterior cruciate ligament injury and functional ankle instability. Gait Posture 2009;29:460-4.

Santos BR, Delisle A, Lariviere C, Plamondon A, Imbeau D. Reliability of centre of pressure summary measures of postural steadiness in healthy young adults. Gait Posture 2008;27:408-15.

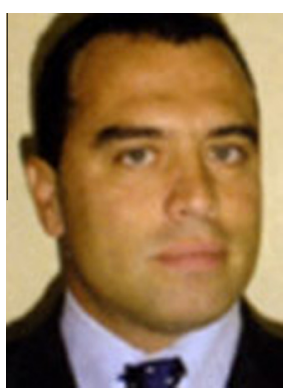

Dr. Alberto Baldini Obtained his degree in 1989 at the University of Milano. He studied at the Post-graduated School of Orthodontics at the University of Milano. He achieved a Ph.D. in Experimental Periodontology at the University of Milano-Bicocca. He is currently attending to his second Ph.D. in Materials for Health Environment and Energy at the University of Rome Tor Vergata with Prof. Paola Cozza. Expert in orthodontics and gnathology, author of articles on journals with Impact Factor.

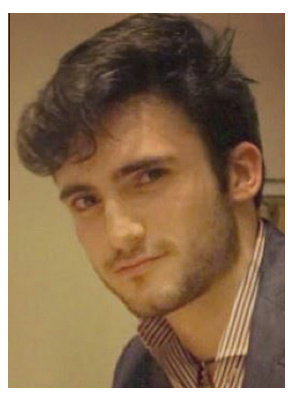

Dr. Alessandro Nota graduated in Dentistry cum Laude in 2011 at the University of Milano-Bicocca. He's currently attending an MSc in Orthodontics at the University of Rome Tor Vergata with Prof. Paola Cozza. His thesis was on the use of the force platform in the analysis of the correlation between dental occlusion and posture. Author of articles in these fields.

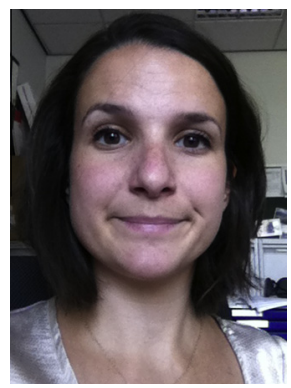

Dr. Valentina Assi During September 2010 started her Ph.D. studentship in Epidemiology at the Wolfson Institute of Preventive Medicine, Queen Mary, University of London. She has a background in experimental and theoretical statistics, and graduated from the Università degli Studi di Milano with a MSc thesis on loglinear symmetry models. Her current research project is supervised by Professor Stephen Duffy and Dr. Jane Warwick and focuses on the properties of mammographic density as a biomarker in breast cancer treatment and prevention, and the potential for automated measurement of breast density.

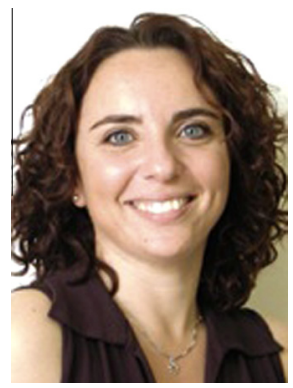

Dr. Fabiana Ballanti Obtained her degree in 2000 at the University of Rome Tor Vergata. She studied at the School of Orthodontics at the University of Rome La Sapienza and is currently an expert in orthodontics. She is attending a Ph.D. in Materials for Health Environment and Energy at the University of Rome Tor Vergata. Actually adjunct Professor in Orthodontics at the University of Rome Tor Vergata. Author of many articles about orthodontics. 


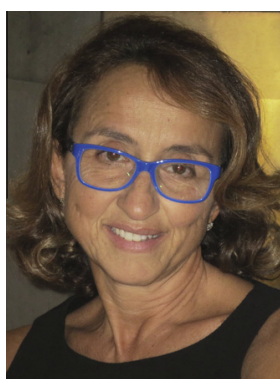

Prof. Paola Cozza is Dean of the School of Dentistry at the University of Rome "Tor Vergata", Director of the Post-graduated School in Orthodontics and Chairman of the Department of Orthodontics at the same University. She has been published extensively on journals with Impact Factor, on the American Journal of Orthodontics and Dentofacial Orthopedics, on the Angle Orthodontist, and on the European Journal of Orthodontics. Prof. Cozza is a member of the Italian Society of Orthodontics (S.I.D.O.), Italian Society of Pediatric Dentistry (S.I.O.I.), European Orthodontic Society (E.O.S.), and World Federation of Orthodontists (W.F.O.) 